\title{
Utilização da análise de FOURIER no estudo de variáveis micrometeorológicas de uma floresta de transição do norte de Mato Grosso
}

\author{
Mariele Regina PINHEIRO ${ }^{1} \&$ Sérgio Roberto de PAULO²
}

\begin{abstract}
RESUMO
Este trabalho foi realizado utilizando dados coletados em uma Floresta de Transição, em uma área pertencente à Fazenda Maracaí no Noroeste de Sinop, MT, com dados micrometeorológicos obtidos com o sistema de correlação de vórtices turbulentos (Eddy Covarience) instalado numa torre de 40 metros. Teve como objetivo principal estudar as potencialidades da análise de Fourier aplicada a dados de fluxo de Calor Latente (LE), Calor Sensível (H) e Temperatura (T). Para os cálculos foram feitas médias de 3 em 3 horas para cada mês, ao longo do período de 1999 a 2005, para as variáveis estudadas. Os períodos dominantes encontrados foram de 24; 12; 4 e 3,4 horas. Os dois primeiros são atribuíveis ao movimento de rotação da Terra, ou seja, à periodicidade dia/noite. Quanto aos dois períodos menores, há indícios que estão relacionados com a dinâmica de abertura dos estômatos. Assim sendo, os resultados deste trabalho indicam que os fatores que influenciam predominantemente as variáveis microclimatológicas durante o dia (freqüências entre $10^{-5}$ a $10^{-4} \mathrm{~Hz}$ ) são a radiação solar e a dinâmica de abertura dos estômatos, um resultado que destaca as potencialidades da aplicação do método de Fourier no estudo da dinâmica de microclimas em ecossistemas.
\end{abstract}

PALAVRAS-CHAVE: Séries de Fourier. Freqüência de Fenômenos Naturais. Radiação Solar. Dinâmica Estomatal.

\section{Use of FOURIER analysis for studying micrometeorological variables of a northern Mato Grosso transition forest}

\section{ABSTRACT}

In this work, we employed data collected in a transition forest, on the Maracaí farm,=northwest of Sinop, MT, Brazil. The data was obtained by the eddy covariance method, using equipment installed on a $42 \mathrm{~m}$ high tower. Its main purpose was to study the potentialities of Fourier analysis applied to data of latent $(\mathrm{H})$ and sensible $(\mathrm{Le})$ heat flux and the air temperature $(\mathrm{T})$. We investigated the main frequencies presented by the data, = and obtained mean values for the variables corresponding to every 3 hours, between 1999 and 2005. The main periods obtained with the Fourier method were 24; 12; 4 and 3.4 hours. The first two are attributed to the solar radiation and to the Earth rotation. The last two periods, as indicated by the data, are related to stomata dynamics. In this way, the results indicate that the main factors that predominantly influence the microclimatological variables during the day (frequencies between $10^{-5} \mathrm{a} 10^{-4} \mathrm{~Hz}$ ), were the solar radiation and the stomata dynamics. These results reinforce the importance of employing the Fourier method in studying microclimatic dynamics of ecosystems.

KEYWORDS: Fourier Series. Frequencies of Natural Phenomena. Solar Radiation. Stomatic Dynamics.

1 UFMT- Universidade Federal de Mato Grosso. marielepinheiro@gmail.com

2 Universidade Federal de Mato Grosso. sergio@cpd.ufmt.br 


\section{INTRODUÇÃO}

Os impactos nas condiçôes e na variabilidade climática, bem como os cenários de mudanças climáticas vêm sendo estudados por vários autores como Fearnside (2006); Marengo e Nobre (2005); Fish et al. (1996); Vourlitis et al. (2008), buscando uma melhor compreensão sobre perturbaçóes e fenômenos naturais intensos que comprometem, freqüentemente, o equilíbrio entre o ambiente social e o ambiente natural.

As emissóes dos gases de efeito estufa vêm aumentando a cada ano. O relatório do IPCC (Intergovernamental Panel on Climate Change) de 2007 diz que, até o fim deste século, a temperatura da Terra pode subir de $1,8^{\circ} \mathrm{C}$ a $4^{\circ} \mathrm{C}$, e prevê o aumento na intensidade de tufóes e secas, além de elevação no nível dos oceanos.

Emissôes de gases do efeito estufa, provocadas pela mortalidade da floresta devido à mudança de clima, fazem parte de uma relaçáo de retroalimentaçáo positiva em potencial que conduz, cada vez mais, ao aquecimento e mais mortalidade (Fearnside 2003).

O conhecimento das componentes envolvidas na interação entre a biosfera e a atmosfera é de extrema importância para a previsão da evolução do clima e da sustentabilidade do ecossistema como um todo e, devido a sua extensão, a Floresta Amazônica tem um importante papel no balanço global, de calor, umidade e de carbono (Oliveira, et al. 2004 apud Fitzjarrald e Moore 1990).

Segundo Platt e Denman (1975), a característica de um sistema náo linear é sua periodicidade, a tendência a um período (ciclo) em relação ao tempo, ao espaço, ou a ambos. De acordo com este ponto de vista, podemos considerar o ecossistema como um sistema não linear, acoplado por um conjunto de diferentes funçóes (Op.Cit., 1975). O elemento básico das séries temporais é o ciclo: uma completa descrição do sistema que deve conter as freqüências de todos os seus ciclos dominantes (Oliveira e Favero 2002). Ou seja, uma maneira de especificar um sistema pode ser a constataçáo de possíveis freqüências de um processo organizado de acordo com a sua relativa importância ou magnitude.

Uma etapa importante do processo de compreensão da dinâmica que governa as variáveis microclimatológicas de um ecossistema é a identificação das principais freqüências (ou freqüências dominantes) que caracterizam a periodicidade da variação temporal dessas variáveis (Baldocchi et al. 2001).

Este trabalho tem como objetivo principal estudar as potencialidades da análise de Fourier aplicada a dados micrometeorológicos (calor latente, calor sensível e temperatura), verificando quais as suas freqüências dominantes. A pesquisa foi realizada utilizando dados coletados em uma Floresta de Transição, no Noroeste de Sinop, MT, nos períodos de 1999 a 2005, obtidos com o método dos vórtices turbulentos por equipamentos (anemômetro sônico e analisador de gases) instalados numa torre de 40 metros.

\section{MATERIAL E MÉTODOS}

\section{Descrição da área em estudo}

Foram utilizados os dados coletados na Amazônia Legal, em uma área pertencente à Fazenda Maracaí, com cerca de 20 $\mathrm{km}^{2}$, localizada na Região Centro-Oeste, a aproximadamente $50 \mathrm{~km}$ no Noroeste de Sinop, Mato Grosso, Brasil ( $11^{\circ} 24.75^{\prime} S$; 55⒚50'O), em uma Floresta de Transição (figura 1) onde está instalada uma torre metálica, de 40 metros, com equipamentos de coletas de dados micrometeorológicos, pertencente ao projeto Experimento de Grande Escala da Biosfera Atmosfera na Amazônia (LBA).

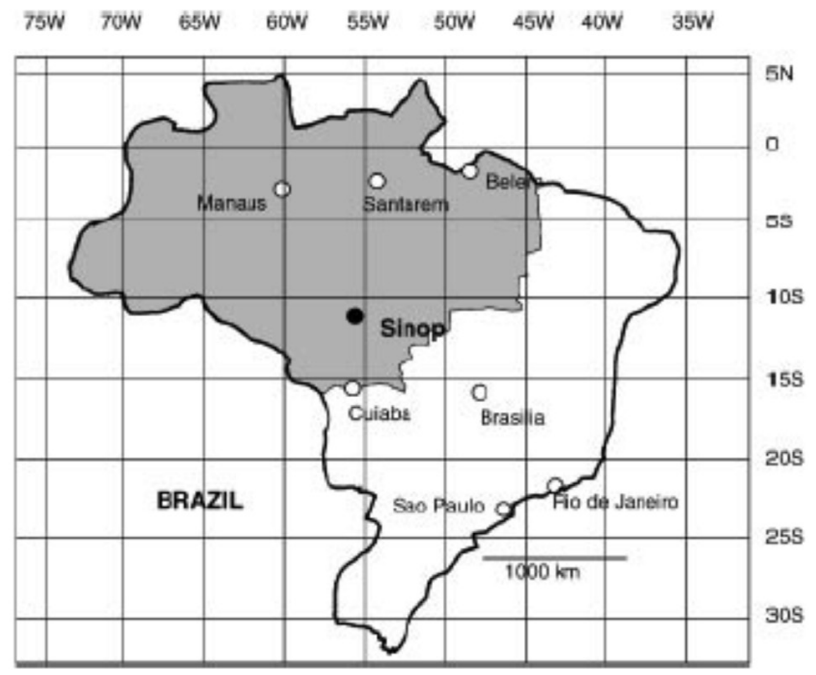

Figura 1 - Local do estudo

Fonte: Vourlitis (2001)

A área de estudo é constituída por uma Floresta Tropical de Transição, com um dossel contínuo, composto de árvores de 28 a 30 m de altura, a 423 metros do nível médio do mar (Vourlitis et al. 2005). O solo é tem alta porosidade com isso drena rapidamente a água da chuva. A temperatura média anual na área de Sinop é de $24^{\circ} \mathrm{C}$ com pequena variação nas estaçôes, e uma precipitação média anual de $2000 \mathrm{~mm}$ com quatro meses de estação seca entre os meses de junho a setembro (Vourlitis et al., 2002).

\section{Metodologia}

Os dados micrometeorológicos foram adquiridos através do sistema de correlação de vórtices turbulentos (eddy covariance), os sensores foram montados a uma altura de $42 \mathrm{~m}$ acima do nível do solo ou 12-14 m sobre o topo da floresta, usado para calcular o fluxo de Calor Latente $(L E)$ e Calor Sensível $(H)$. 
Os valores medidos dos fluxos de Calor Latente $(L E)$, Calor Sensível $(H)$, foram determinados usando o método de correlação de vórtices turbulentos, que determina os fluxos, calculando a correlação entre os desvios temporais de alta freqüência da temperatura do ar (fluxo de calor sensível) e da umidade do ar (fluxo de calor latente.

De 1999 a 2005, o sistema de correlação de vórtices turbulentos era constituído por um Anemômetro Sônico (Applied Technologies, Inc., Longmont, CO, USA), que quantifica a velocidade do vento e a temperatura nos três eixos ortogonais e um Psicrômetro modelo (HMP45C, Campbell Scientific, Inc.) no qual realiza medidas de temperatura, umidade relativa do ar e por um conversor analógico/digital (datapacker).

O sistema de aquisição de dados era composto por um datalogger (CR 10X, Campbell Scientific, Inc., Ogden, Utah), e um laptop no qual manipulava os dados com um fluxo de respostas rápidas de $10 \mathrm{~Hz}$ utilizando a técnica de médias de 200s com filtragem digital recursiva ( para os fluxos de energia calor sensível e calor latente), armazenava as variáveis como médias de 30 minutos.

Após a reforma realizada na torre em 2005, foi modificado o sistema de correlação de vórtices turbulentos, o anemômetro sônico tridimensional foi substituído por outro (CSAT3: 3-D anemometer sonic, Campbell, USA). Depois da reforma, o sistema de aquisição prescinde da utilização de um computador portátil, pois passou a ser constituído por um datalogger (CR-5000: Campbell, USA). Os dados são analisados em planilhas do software Microsoft Excel.

Nesse estudo foram utilizadas as variáveis: Fluxos Calor Latente $(L E)$ e Calor Sensível $(H)$, e temperatura (T) com dados dos anos de 1999 a 2005. Para análise dos dados será utilizado um método de análise de séries temporais, a Série de Fourier.

Com base na torre e esta técnica micrometeorológica quantifica diretamente as trocas de massa e energia da superfície-atmosfera, pela medida do transporte turbulento de vapor d'água e calor (Vourlitis et. al. 2002; Vourlitis et al. 2001; Verma 1990; Baldocchi et al. 2001).

Os dados brutos coletados na torre micrometeorológica na Floresta de Transiçáo, os dados diários foram acumulados a cada 30 minutos em um datalogger, fazendo uma média de $3 \mathrm{em}$ 3horas começando de 0:00 hora a 23:30 horas, foram trabalhados em planilhas do software Microsoft Excel, sendo separados por variável. Cada variável foi organizada em uma planilha por mês, onde foi calculado a média, de $3 \mathrm{em} 3$ horas.

No tempo em que foi realizado o estudo, houve falhas nos equipamentos, assim alguns dias ou meses tiveram ausência de dados, com isso os meses com maior ausência de dados foram descartados, sendo por volta de 50 meses.

\section{Séries de Fourier}

Uma série temporal pode ser definida como um conjunto de observaçóes de uma variável dispostas seqüencialmente no tempo. Normalmente as séries temporais são analisados a partir de seus principais movimentos descritos como: tendência, ciclo, sazonalidade e variaçôes aleatórias (Oliveira e Favero 2003).

A série de Fourier envolve a representações a funções periódicas mais ou menos complexas em termos de funções simples, como seno e coseno. A análise de Fourier é uma das formas mais tradicionais para tratamento de sinais e séries temporais. Esta técnica foi criada por Jean Baptiste Joseph Fourier e publicada em 1822 no seu trabalho intitulado Thèorie Analitique de la Chaleur. Fourier dedicou-se na resoluçâo das equaçôes diferenciais que regem a transferência de calor utilizando uma técnica de séries de senos e cossenos (Série de Fourier) para resolver seus problemas (Morettin e Toloi 2006).

$$
f(x)=\frac{A_{0}}{2}+\sum_{n=1}^{\infty} a_{n} \cos n \cdot x+\sum_{n=1}^{\infty} b_{n} \operatorname{sen} . n . x \quad \text { Equação I }
$$

Os coeficientes $A_{0}, a_{n} e b_{n}$ são relacionadas com funçóes $\operatorname{de} f(\mathrm{x})$ definidas por integrais. A priori, náo temos o interesse em aprofundar nas deduções de séries de Fourier, assim apresentaremos os coeficientes da função acima.

- $\mathrm{a}_{0}$ corresponde ao valor médio dos dados micrometeorológicos;

- $\quad a_{n}$ e b são coeficientes que satisfazem as condiçôes de Dirichlet e usadas em relaçóes ortogonais, no qual validam a teoria de Sturm- Liouville.

$$
\begin{array}{lll}
a_{n}=\frac{1}{\pi} \int_{0}^{2 \pi} f(t) \cos n t a t & ; & \text { Equação II } \\
b_{n}=\frac{1}{\pi} \int_{0}^{2 \pi} f(t) \text { senntdat } & ; & \text { Equação III }
\end{array}
$$

As condições impostas para a função $f(\mathrm{x})$ na equação I ser válida são que a função $f(\mathrm{x})$ deve ser contínua, embora seja possível obter representaçóes satisfatórias no caso de funçōes descontínuas.

\section{Vantagens da Utilização da Série de Fourier}

\section{- Função Descontínua:}

A Série de Fourier tem a possibilidade de representação de uma série descontínua, na qual não precisa ter todos os dados da pesquisa para obter um resultado real. 


\section{- Função Periódica}

Se $f(x)$ tiver um período de $2 \pi$, pode-se expandir a série a um período de $2 \pi, 2 \pi / 2,2 \pi / 3$, isto garante a periodicidade de $f(x)$ para um intervalo de $[0,2]$ ou $[-\pi, \pi]$, para todo $\mathrm{x}$ finito.

Utilizando a relaçáo, considera-se a propriedade de simetria. Usando o intervalo $[-\pi, \pi]$, sin x é impar e cos x é uma função par de x. Pelas eq. II e III, se f (x)é constante $a_{n}$ será 0 se $f(x)$ for impar, todo $b_{n}=0$. Ou seja

$$
\begin{aligned}
& f(x)=\frac{a_{0}}{2}+\sum_{n=1}^{\infty} a_{n} \cos n x, \text { par, } \\
& f(x)=\sum_{n=1}^{\infty} b_{n} \sin n x, \text { impar. }
\end{aligned}
$$

Equação IV

Equação V

Estas propriedades podem ser expandidas em uma dada função.

\section{- Mudança de intervalo}

$\mathrm{Na}$ pesquisa restringiu-se o comprimento do intervalo a $2 \pi$. Se $f(x)$ tiver um período de $2 \mathrm{~L}$, pode-se escrever:

$f(x)=\frac{a_{0}}{2}+\sum_{n=1}^{\infty}\left[a_{n} \cos \frac{n \pi x}{L}+b_{n} \sin \frac{n \pi x}{L}\right]$.

Equação VI

com

$a_{n}=\frac{1}{L} \int_{-L}^{L} f(t) \cos \frac{n \pi t}{L} d t, \mathrm{n}=0,1,2,3 \ldots$,

Equação VII

$b_{n}=\frac{1}{L} \int_{-L}^{L} f(t) \sin \frac{n \pi t}{L} d t, \mathrm{n}=0,1,2,3 \ldots$,

Equação VIII

substituindo x na Eq. I com $\pi \mathrm{x} / L$ e t nas Eq. II e I $\operatorname{com} \pi t / L$. Para $\mathrm{f}(\mathrm{x})$ periódico com um período de $2 \mathrm{~L}$, todo o intervalo fará $\left(\mathrm{x}_{0}, \mathrm{x}_{0}+2 L\right)$.

\section{RESULTADOS E DISCUSSÃO}

A análise da Série de Fourier foi realizada para cada mês de cada variável (Calor Sensível, Calor Latente e Temperatura), entre agosto de 1999 e dezembro de 2005. Primeiramente foram obtidos os valores de An e Bn conforme as equações 2 e 3 em seguida os gráficos das médias mensal (An e Bn), através dos gráficos foram achados os períodos principais.

O eixo vertical dos gráficos (fig. 2, 3 e 4) representa os coeficientes $\mathrm{An}$ e Bn, que indicam com que intensidade a série temporal correspondente aos dados varia em cada freqüência específica. Através dos gráficos, pode-se perceber características tanto comuns como distintas, em relação à posição dos picos e das lacunas de cada variável.
Por exemplo, na figura 2a, que se refere ao Fluxo de Calor Latente, tem-se 4 picos $(\mathrm{n}=31 ; 62 ; 186 ; 217)$. Considerando-se que se trata de um mês com 31 dias, os períodos correspondentes podem ser calculados pela relação $31 / \mathrm{n}$, o que resulta em 1 dia, 12 horas, 4 horas e 3,43 horas, respectivamente.

Os picos dos Fluxos do Calor Sensível e Temperatua, na figura 3, como aqueles mostrados na figura 2, têm períodos de 1 dia, 12 horas, 4 horas e 3,45 horas, respectivamente. Além desses, no caso do calor sensível, Bn apresenta mais 2 picos nos períodos de $8 \mathrm{~h}$ e $4,96 \mathrm{~h}$.

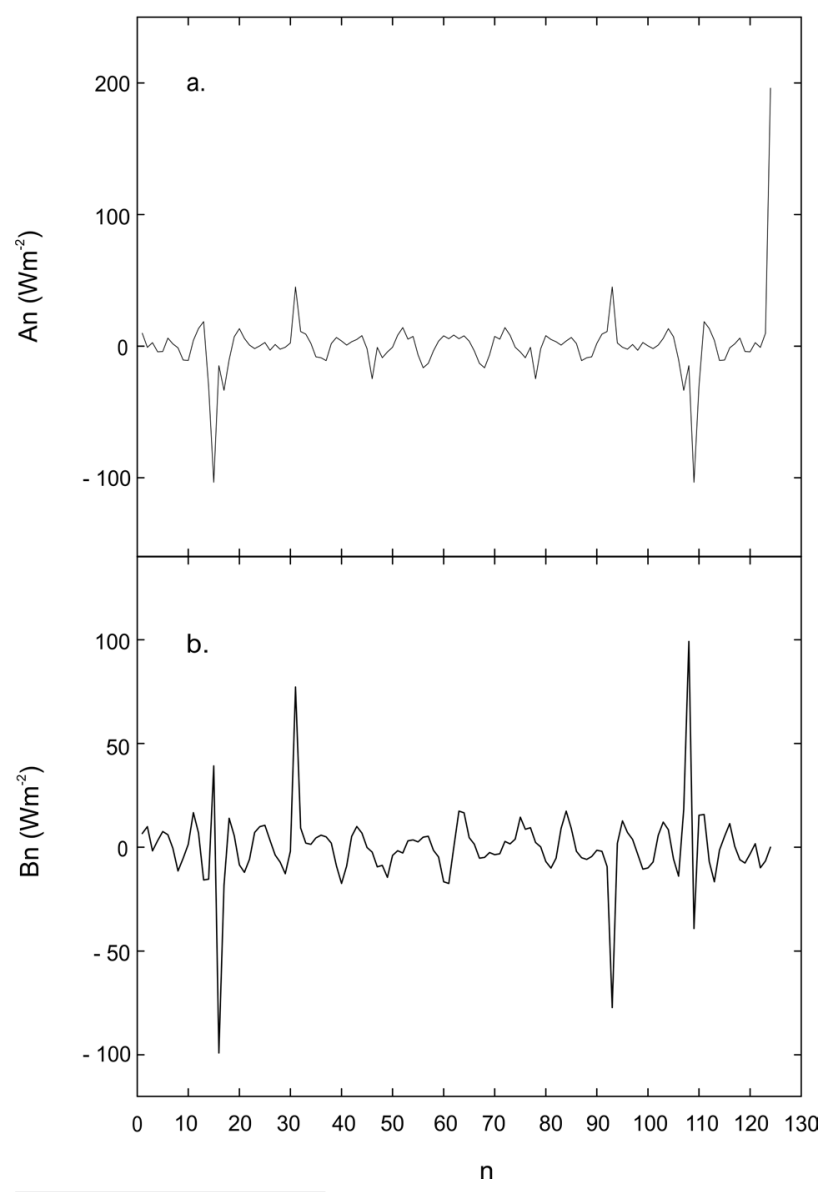

Figura 2 - Valores de An em a e Bn em $\boldsymbol{b}$, para o Fluxo do Calor Latente, em julho de 2002

Numa análise de Fourier, cada pico pode representar um fenômeno diferente. Adiante será analisado o significado de cada um deles, mas fica mais ou menos evidente que o pico relacionado a 1 dia se refere ao ciclo diário com alternância de dias e noites, consequentemente fazendo com que os fluxos de calor tenham máximos que se repetem em ciclos diários.

As características dos gráficos para temperatura, figuras 3 c e d, mostram 2 picos mais pronunciados para An e Bn com 

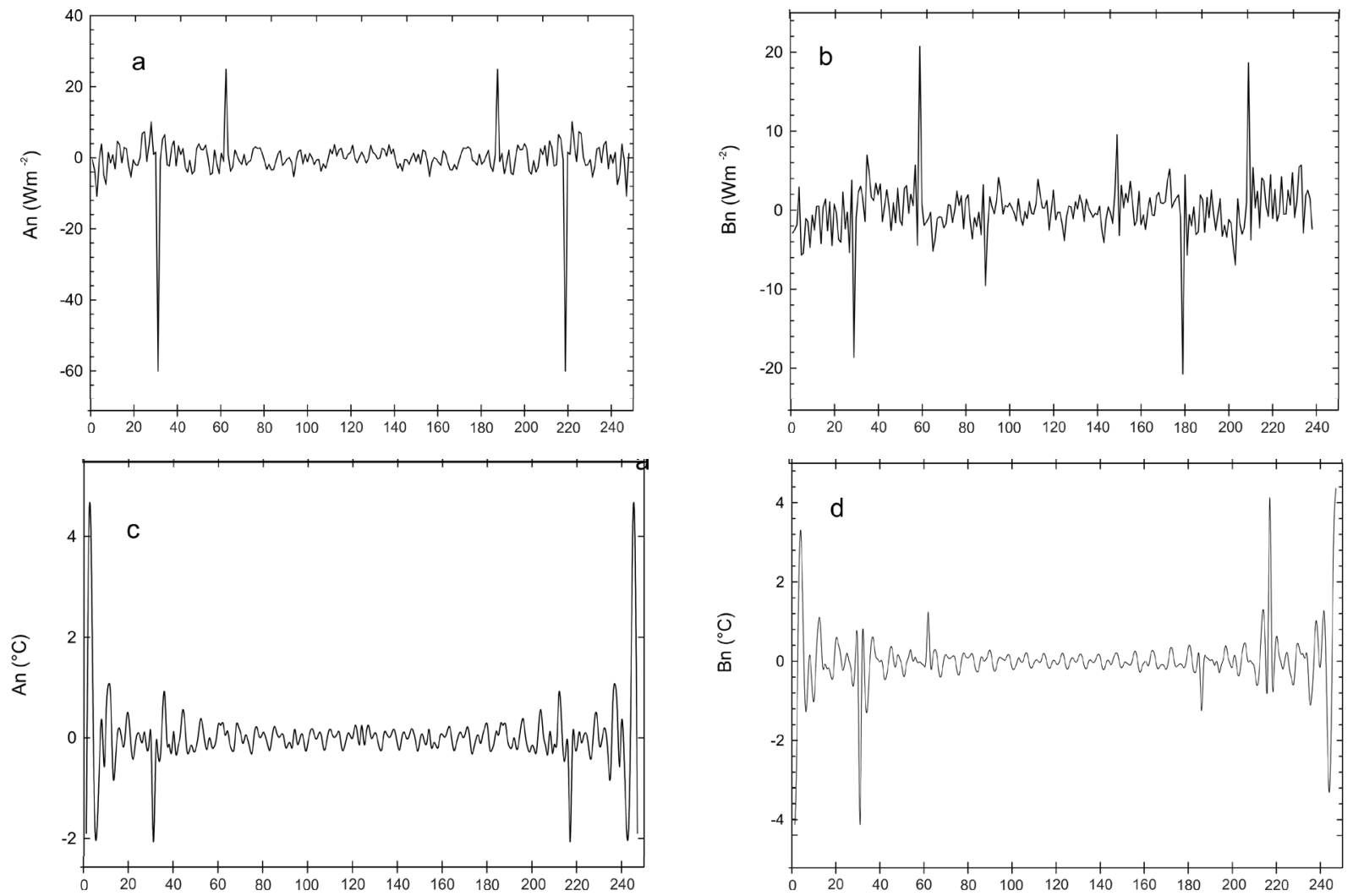

Figura 3 - Valores de An em a e Bn em b, para o Fluxo do Calor Sensível. $\boldsymbol{c}$ e $\boldsymbol{d}$ referem-se respectivamente ao An e Bn para a Temperatura, todos em maio de 2001.

Tabela 1 - Valores dos períodos, para o fluxo de Calor Latente, em relação ao An e Bn entre agosto de 1999 a dezembro de 2005

\begin{tabular}{|c|c|c|c|c|c|c|c|c|c|c|c|c|}
\hline \multicolumn{13}{|c|}{ CALOR LATENTE } \\
\hline Mês & $t(d)$ & $\begin{array}{c}\mathrm{n}^{0} \\
\text { dados }\end{array}$ & An / Período & $\begin{array}{c}\text { An / } \\
\text { Período }\end{array}$ & An / Período & An / Período & Bn / Período & $\begin{array}{c}\text { Bn / } \\
\text { Período }\end{array}$ & $\begin{array}{c}\mathrm{Bn} / \\
\text { Período }\end{array}$ & $\begin{array}{c}\text { Bn / } \\
\text { Período }\end{array}$ & $\begin{array}{c}\mathrm{Bn} / \\
\text { Período }\end{array}$ & $\begin{array}{c}\text { Bn / } \\
\text { Período }\end{array}$ \\
\hline ago/99 & 18,3 & 132 & $52,01 / 1,12 d$ & $21,9 / 12 h$ & $99 / 4,38 h$ & $52,01 / 4 h$ & $28,3 / 1,12 d$ & $-13,4 / 12 h$ & & $13,4 / 4,38 h$ & $-28,3 / 4 h$ & \\
\hline set/99 & 30 & 240 & $-41,3 / 1 d$ & $22,4 / 12 h$ & $22,4 / 4 h$ & $-41,3 / 3,43 h$ & $28,3 / 1 d$ & $-15,3 / 12 \mathrm{~h}$ & & & $15,3 / 4 h$ & $28,3 / 3,43 \mathrm{~h}$ \\
\hline out/99 & 31 & 248 & $-82,4 / 1 d$ & $62 / 12 \mathrm{~h}$ & $186 / 4 h$ & $-82,4 / 3,43 h$ & $-40,2 / 1 d$ & $30,2 / 12 \mathrm{~h}$ & & & $-30,2 / 4 h$ & $40,2 / 3,43 \mathrm{~h}$ \\
\hline $\mathrm{jan} / 01$ & 31 & 248 & $-81,4 / 1 d$ & & & $-81,4 / 3,43 h$ & $-52,9 / 1 d$ & $35,4 / 12 h$ & & & $-35,4 / 4 h$ & $52,9 / 3,43 \mathrm{~h}$ \\
\hline $\mathrm{fev} / 01$ & 29 & 232 & $-132,4 / 1 d$ & $35 / 12 h$ & $35 / 4 h$ & $-132,4 /$ & $-81,2 / 1 d$ & $63 / 12 \mathrm{~h}$ & & & $-63 / 4 h$ & $43 \mathrm{~h}$ \\
\hline $\mathrm{mar} / 01$ & 30 & 240 & $26 / 1 d$ & $5,8 / 12 \mathrm{~h}$ & $35,8 / 4 h$ & $43 \mathrm{~h}$ & $-78,6 / 1 d$ & $61,5 / 12 h$ & $-15,3 / 8 h$ & $15,3 / 4,96 h$ & $-61,5 / 4 h$ & $78,6 / 3,43 \mathrm{~h}$ \\
\hline $\mathrm{abr} / 01$ & 30 & 240 & $-161,8 / 1 d$ & $49,9 / 12 h$ & $49,9 / 4 h$ & $-161,8 / 3,43 h$ & $-13,3 / 1 d$ & $13,7 / 12 \mathrm{~h}$ & & & $-13,7 / 4 h$ & $13,3 / 3$ \\
\hline mai/01 & 31 & 248 & 101,8/1d & $37,4 / 12 h$ & 3 & -101 & $d$ & $50,5 / 12 h$ & $8 \mathrm{~h}$ & $\begin{array}{l}19,7 / \\
4,96 h\end{array}$ & $-50,5 / 4 h$ & 5 \\
\hline jun/02 & 30 & 240 & $-93,2 / 1 d$ & $26,4 / 12 h$ & $26,4 / 4 h$ & $-93,2 / 3,43 h$ & $-59,8 / 1 d$ & $55,6 / 12 \mathrm{~h}$ & $-18,2 / 8 h$ & $18,2 / 4,96 h$ & $-55,6 / 4 h$ & $59,8 / 3,43 h$ \\
\hline $\mathrm{jul} / 02$ & 31 & 248 & $-67,5 / 1 d$ & $28,3 / 12 h$ & $28,3 / 4 h$ & $67,5 / 3,43 \mathrm{~h}$ & $-49,6 / 1 d$ & $38,6 / 12 h$ & & & $-38,6 / 4 h$ & $49,6 / 3,43 \mathrm{~h}$ \\
\hline ago/02 & 29,7 & 238 & $9 / 1 d$ & $45,8 / 12 h$ & $45,8 / 4 h$ & $3,43 \mathrm{~h}$ & $-104,4 / 1 d$ & $42,8 / 12 h$ & & & $-42,8 / 4 h$ & $\begin{array}{l}104,4 / \\
3,43 \mathrm{~h}\end{array}$ \\
\hline $\mathrm{abr} / 05$ & 29,5 & 238 & $-72,2 / 1 d$ & $-26,4 / 12 h$ & $-26,4 / 3,93 h$ & $-72,2 / 3,35 \mathrm{~h}$ & $1,4 / 1 d$ & $40,4 / 12 h$ & 15,7/ 8h & $\begin{array}{l}-15,7 / \\
4,96 \mathrm{~h}\end{array}$ & $-40,4 / 4 h$ & $41,4 / 3,43 h$ \\
\hline mai/05 & 25,5 & 204 & $-67,9 / 1 d$ & $18,9 / 12 h$ & $18,9 / 4 \mathrm{~h}$ & $-67,9 / 3,43 h$ & $-78,9 / 1 d$ & $66,6 / 12 \mathrm{~h}$ & & & $-66,6 / 4 h$ & $78,9 / 3,43 \mathrm{~h}$ \\
\hline out/05 & 31 & 248 & $-104,7 / 1 d$ & $38,8 / 12 h$ & $38,8 / 4 \mathrm{~h}$ & $104,7 / 3,43 \mathrm{~h}$ & $-15,3 / 1 d$ & $17,3 / 12 \mathrm{~h}$ & $-10,9 / 8 h$ & $10,9 / 6 h$ & $-17,3 / 4 \mathrm{~h}$ & $15,3 / 3,43 \mathrm{~h}$ \\
\hline nov/05 & 30 & 240 & $-79,5 / 1 d$ & $29,4 / 12 h$ & $29,4 / 4 h$ & $-79,5 / 3,43 \mathrm{~h}$ & $-54,3 / 1 d$ & $28,8 / 12 h$ & & & $-28,8 / 4 h$ & $54,3 / 3,43 \mathrm{~h}$ \\
\hline $\mathrm{dez} / 05$ & 28,5 & 228 & $52 / 1 d$ & $-21,9 / 12 h$ & $-21,9 / 4 h$ & $52 / 3,43 \mathrm{~h}$ & $55,3 / \quad 1 d$ & $41,8 / 12 h$ & & & $-41,8 / 4 \mathrm{~h}$ & $55,3 / 3,43 \mathrm{~h}$ \\
\hline
\end{tabular}


$\mathrm{n}=30$ e 210. Calculando os períodos, sấo obtidos valores correspondentes a $1 \mathrm{~d}$ e $3,43 \mathrm{~h}$, respectivamente. Para Bn tem-se outros dois picos em $n=60$ e 180, correspondendo a $12 \mathrm{~h}$ e $4 \mathrm{~h}$.

$\mathrm{Na}$ tabela 1 são mostrados valores de $\mathrm{An}$ e $\mathrm{Bn}$ correspondentes aos picos mais pronunciados da análise de Fourier do fluxo de calor latente, obtidos para dados de diversos meses. Os períodos predominantes correspondem a 1d, 12h, 4h e 3,43h, tanto para o An como para o Bn. Em cinco meses apareceram picos para $\mathrm{Bn}$ correspondentes a $8 \mathrm{~h}$ e 4,96h.

\section{Explicação Teórica de cada coeficiente}

Para formular uma explicação física para cada freqüência dominante encontrada, na figura 4 são mostrados os comportamentos de alguns dos termos da série de Fourier correspondente aos dados no período de um dia (24 horas), utilizando-se os valores dos coeficientes relativos ao fluxo de calor latente para julho de 2002. A figura 4a, referente ao termo correspondente a $\mathrm{A} 30\left(A_{30} \cos \left(\frac{30 \pi t}{L}\right)\right)$, mostra que o valor das variáveis microclimatológicas têm maior valor na metade do dia. Como é conhecido que os valores de temperatura e fluxos de calor são máximos (genericamente falando) quando a radiação líquida incidente é máxima, segue-se que o comportamento desse termo deve-se ao ciclo diário da incidência de radiaçấo solar, ou seja, o período de 24 horas deve-se à radiaçáo solar.

Já na figura $4 \mathrm{~b}$ é mostrada a soma dos termos correspondentes a $\mathrm{A} 30$ e $\mathrm{A} 60\left(A_{30} \cos \left(\frac{30 \pi t}{L}\right)+A_{60} \cos \left(\frac{60 \pi t}{L}\right)\right)$ - períodos de 24 e 12 horas. Pode-se notar que, com a introduçáo do termo A60, o valor da curva fica ainda mais pronunciado durante o meio do dia e mais próximo de zero à noite. Assim sendo, o período de $12 \mathrm{~h}$, na análise de Fourier, pode ser atribuído à tendência da série em reproduzir o fato de que os fluxos de calor são próximos de zero à noite (ou a temperatura menor à noite que no dia), ou seja, existe uma assimetria nos valores das variáveis com a relação simétrica dos senos e cossenos (os dados apresentam um pico positivo durante o dia, mas não apresentam um pico negativo durante a noite).

Já uma explicação física para os coeficientes Bn pode ser obtida se somarmos os termos correspondentes aos coeficientes $\mathrm{A} 30$ e $\mathrm{B} 30\left(A_{30} \cos \left(\frac{30 \pi t}{L}\right)+B_{30} \operatorname{sen}\left(\frac{30 \pi t}{L}\right)\right)$, figura 4c. Pode-se observar um deslocamento para a direita em relaçáo à figura 4a. Com isso, para o Calor Latente, pode-se afirmar que existe uma tendência de que o fluxo do calor latente na segunda metade do dia (das 12 as $18 \mathrm{hs}$ ) seja um pouco maior que na primeira metade do dia (das 7 as $12 \mathrm{hs}$ ), caso o coeficiente B30 for positivo, e um pouco menor que na primeira metade do dia, caso o coeficiente B30 seja negativo (observar que a maioria dos valores de B30 - correspondentes a 1 dia - apresentados na Tabela I são negativos). Possivelmente, isso se deve à dinâmica de abertura dos estômatos. Vários fatores como água, luz, $\mathrm{CO}_{2}$ e temperatura influenciam o mecanismo estomático de abertura e fechamento. As células estomáticas são capazes de realizar fotossíntese, assim as plantas precisam abrir os estômatos para absorção de $\mathrm{CO}_{2}$, mas também necessitam fechá-los para evitar a perda de água. A regulação temporal da abertura estomática funciona da seguinte maneira, à noite quando não há fotossíntese a abertura estomática fica pequena, nas manhãs ensolaradas com água abundante e quando a radiação solar incidente na folha favorecendo altas taxas de fotossíntese, a demanda por $\mathrm{CO}_{2}$ dentro da folha é alta e por isso o poro estomático permanece amplamente aberto. Por volta do meio-dia os estômatos se fecham, pelo aumento de radiação.

A figura 4d, foi produzida através da soma de todos os termos significativos da série de Fourier (correspondente aos coeficientes An e Bn com períodos de 24; 12; 4 e 3,4 horas), durante um dia. De acordo com o perfil do gráfico, o fluxo do calor latente durante a noite tende a ser próximo de zero e diferente de zero durante o dia. Os "zig-zags" do gráfico devem-se ao fato de que a série de Fourier, conforme já comentado, por se basear em senos e cossenos, náo consegue reproduzir, com um pequeno número de termos, a constância do valor de LE durante a noite (LE, à noite, seria representado por uma função constante e nula).

Ainda segundo o perfil do gráfico, durante o dia, entre os horários da 10:00h às 17:00h, há dois picos para o valor do fluxo de calor latente, separados por um período de menor valor por volta das 14 horas. Tal comportamento pode ser também atribuído à dinâmica de abertura e fechamento dos estômatos. Quando a atmosfera contém pequena quantidade de vapor d'água (baixa umidade), pode haver alta taxa de perda de água (o que pode acontecer no meio do dia). Nessas condiçóes, os estômatos tendem a se fechar para evitar a desidratação. Como esse perfil do gráfico se deve à adição dos termos correspondentes a 4 e 3,4 horas, pode-se explicar esses períodos como decorrência da dinâmica estomatal. Caso isso se confirme, com resultados adicionais de outras pesquisas, a dinâmica estomatal se coloca como o segundo fator mais importante (após a radiação solar) no comportamento das variáveis microclimáticas numa escala de tempo de um dia.

A influência da dinâmica estomatal sobre o fluxo de calor latente (e sua relaçấo com as freqüências correspondentes a períodos em torno de 3 e 4 horas) pode ser compreendida se o perfil de LE ao longo do dia é analisado. Conforme a figura $5 \mathrm{a}$, correspondentes a dados experimentais do fluxo de calor latente no dia 4 de abril de 2001, o perfil de LE durante o dia apresenta variaçóes com subidas e descidas compatíveis com os períodos entre 3 e 4 horas $\left(7\right.$ a 9 $\times 10^{-5} \mathrm{~Hz}$ ), ou seja, a variação temporal de LE durante o dia não é suave. 


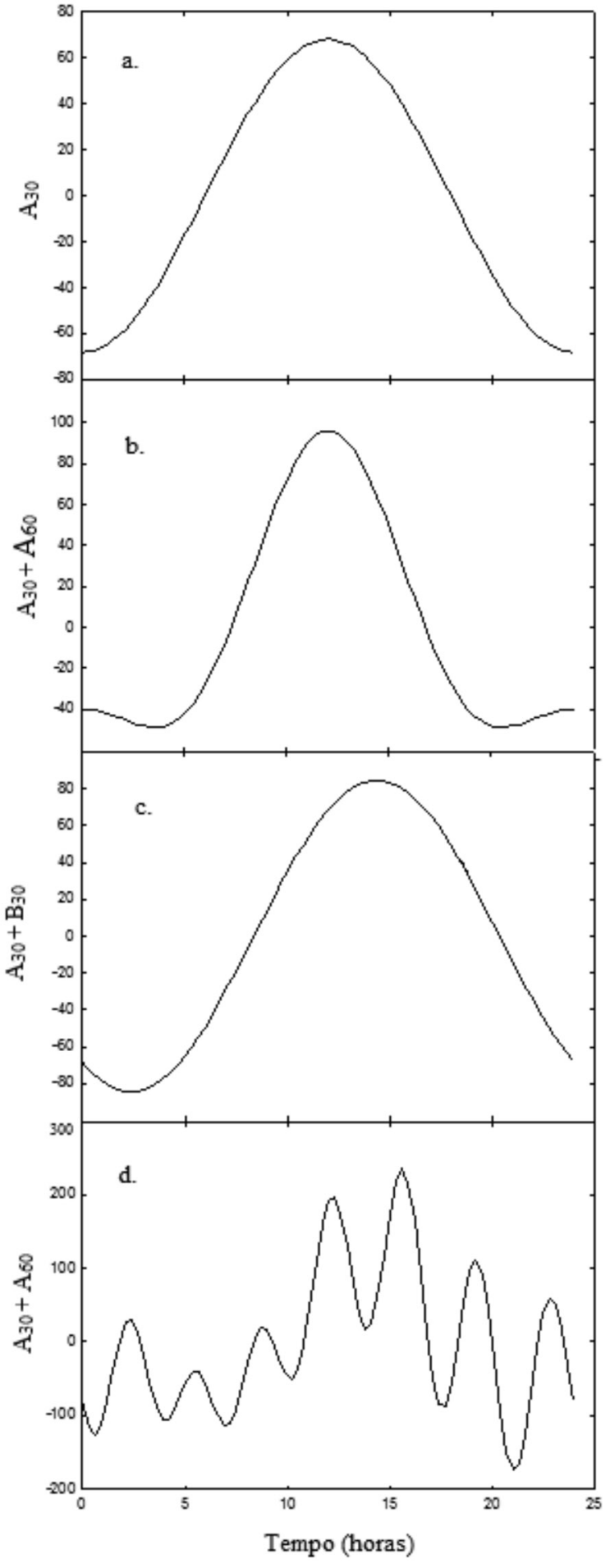

Figura 4 - Comportamento da Série de Fourier, em a considerando-se apenas o termo $A_{30}$, em $\boldsymbol{b}$, a soma dos termos $A_{30}+A_{60}$, em $\boldsymbol{c}$, as somas dos termos $A_{30}$ e $B_{30}$, e em $\boldsymbol{d}$, a soma de todos os termos significativos.
Tal comportamento se deve ao fato de que, ao se abrirem para absorver gás carbônico, os estômatos tendem a perder água para o meio ambiente na forma de vapor. Tal efeito é particularmente crítico nas estaçóes de seca, período em que a biosfera tem que se resguardar da perda de água para a atmosfera. Assim sendo, os estômatos, após se abrirem por um determinado tempo para absorver carbono, fecham-se novamente para estabelecer um controle sobre a perda de água. Desta forma, estabelece-se uma dinâmica intermitente controlada pela entrada de $\mathrm{CO}_{2}$ e saída de $\mathrm{H}_{2} \mathrm{O}$.

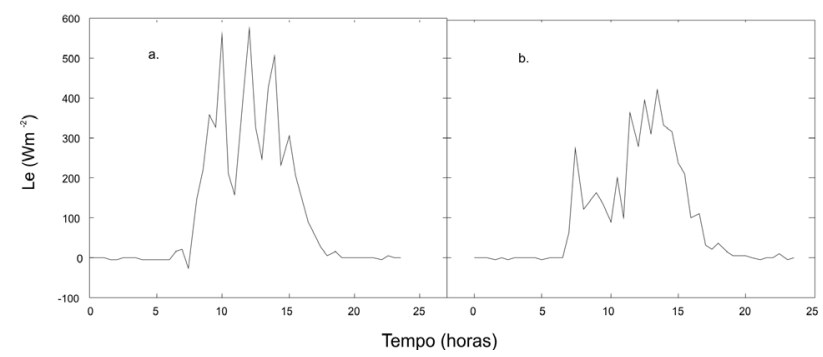

Figura 5 - Fluxo de calor latente em função do tempo nos dias julianos 94 (a) e 109 (b) de 2001.

$\mathrm{O}$ aparecimento de coeficientes $\mathrm{B}_{\mathrm{i}}$ significativos - os quais estabelecem uma assimetria no comportamento das variáveis microclimatológicas - também pode estar relacionado com a dinâmica estomatal, como é indicado pelo comportamento temporal de LE em alguns dias específicos, correspondente à figura $5 b$.

\section{CONCLUSÃO}

Analisando os resultados obtidos pela análise de Fourier dos dados sobre o Fluxo de Calor Latente, Fluxo de Calor Sensível e Temperatura, pode-se concluir que esse instrumento de investigação é capaz de fornecer informaçôes importantes sobre a dinâmica das variáveis microclimatológicas.

Foram encontradas quatro freqüências dominantes para as variáveis estudas numa escala de tempo de um dia: $24 ; 12$; 4 e 3,4 horas. As freqüências dominantes encontradas para o fluxo de Calor Sensível, fluxo de Calor Latente e Temperatura correspondente aos períodos de 1 dia e 12 horas podem ser atribuídos à variação diária da radiação solar, devido ao movimento de rotação da Terra. Já os valores correspondentes a 4 e 3,4 horas podem ser explicados em função da dinâmica dos estômatos.

A se confirmarem tais resultados por outras pesquisas, a dinâmica estomatal se coloca como o segundo fator mais importante para a compreensáo do comportamento das variáveis microclimatológicas durante o dia. Isso não quer dizer que não existam outros fatores igualmente importantes correspondentes a freqüências mais baixas, como por exemplo 
os ciclos anuais e o efeito El Niño. Entretanto, os resultados da análise de

Fourier indicam que considerar a dinâmica estomatal é essencial em quaisquer modelos de descrição das variáveis microclimatológicas, pelo menos na floresta de transição estudada.

\section{AGRADECIMENTOS}

Agradecimento a Coordenação de Aperfeiçoamento de Pessoal de Nível Superior (CAPES), ao Programa de Pósgraduação em Física Ambiental

\section{REFERÊNCIAS}

Baldocchi, D.D.; Falge, E.; Wilson, K. 2001. A spectral analysis of biosphere-atmosphere trace gas flux densities and meteorological variables across hour to multi-year time scales. Agricultural and Forest Meteorology. 107:1-27.

Fearnside, P.M. 2003. A Floresta Amazônica nas Mudanças Globais. Instituto Nacional de Pesquisas da Amazônia-INPA, Manaus, AM. 134pp.

Fearnside, Philip M. 2006. Desmatamento na Amazônia: dinâmica, impactos e controle. Acta Amazônica. 36(3): 395-400.

FISH, G., Marengo , J. Nobre, C.A, 1996. Clima da Amazônia, MCT/INPE/CPTEC- Instituto Nacional de Pesquisas Espaciais, Cach. Paulista SP, 24-41.

Marengo, J.A. \& C.A. Nobre. 2005. Liçóes do Catarina e do Katrina. As mudanças do clima e os fenômenos extremos. Ciência Hoje, 37(221):22-27.

Ministério Da Ciência E Tecnologia - Instituto Nacional de Pesquisas Espaciais- INPE. 2007. Relatório do Painel Intergovernamental sobre Mudança do Clima - IPCC. Disponível em: http://www. cptec.inpe.br/mudancas_climaticas/pdfs. Acesso em 28/10/2007

Morettin, P. A.; Toloi, Clélia, M. C. 2006 Análise de Séries Temporais. São Paulo. Edgard Blucher.415-434
Oliveira, P. J. de, Rocha, E. J. P. da, Fisch, G.; Kruijt, B.; Ribeiro, J. B. M. 2004. Meteorological effects of a cold spell event over Amazonia: a case study. Acta Amazônica 34(4).

Olivera, M. A.; Favero, L.P.L. 2002. Uma breve descrição de algumas técnicas para análise de séries temporais: Séries de Fourier, Wavelets, Arima, Modelos Estruturais para séries de tempos e redes neurais.. São Paulo. EdUSP

Platt,T.; Denmam, K. L. 1975. Spectral Analysis in Ecology. Annual Review of Ecology and Systematics, 6:189-210.

Verma, S. B. 1990. Micrometeorological methods for measuring surface fluxes of massandenergy. Remote Sensing Reviews, 5: 99-115.

Vourlitis, G. L.; Nogueira, J. De S., Priante Filho, N.; Hoeger, W.; Raiter,F.; Biudes, M. S.; Arruda, J. C.; Capistrano, V. B.; De Faria, J. L. B.; Lobo, F.de A. 2005.The sensitivity of diel $\mathrm{CO}_{2}$ and $\mathrm{H}_{2} \mathrm{O}$ vapor exchange of a tropical transitional forest to seasonal variation in meteorology and water availability. Earth Interactions, 9: 27-35.

Vourlitis, G.L. ; Nogueira, J. S.; Lobo, F.A., Sendal, K. R; Paulo, S.R. de; Dias, C.A.; Pinto Jr, O. B.; Andrade, N. L. 2008. Energy balance and canopy conductance of a tropical semi-deciduous forest of the southern Amazon Basin. Water Resources Research, 44:W03412.

Vourlitis, G.L.; Priante Filho, N.; Hayashi, M. M.; Nogueira, J. S. de; Caseiro, F.; Campelo Jr., J. H. 2001. Seasonal variations in the net ecosystem $\mathrm{CO}_{2}$ exchange of a mature Amazonian transitional tropical forest (cerradáo). Functional Ecology, 15: 388-395.

Vourlitis, G.L.; Priante Filho, N.; Hayashi, M. M.; Nogueira, J. S. De; Hoeger, W.; Raiter, F.; Campelo Jr., J. H. 2002. The Role Of Seasonal variations in meteorology on the net $\mathrm{CO}_{2}$ exchange of a Brazilian transitional tropical forest. Water Resources Research, 38(6) 30-1-30-11

Recebido em 31/12/2009

Aceito em 20/04/2010 